\title{
Detección de ZONAS DE PENUMBRA Y VISIBILIDAD A LOS FLU.JOS ELECTROMAGNÉTICOS EN CAMPOS ABIERTOS
}

\author{
Jairo Madrigal Argáez ${ }^{1}$ \\ Manuel Julio GarCía Ruiz ${ }^{2}$
}

\section{Resumen}

Este artículo exhibe un procedimiento computacional que permite explorar, sobre un mapa de elevación del terreno (DTM) de una región geográfica de interés, las zonas que están en la línea de incidencia directa de los flujos de energía generados por fuentes puntuales localizadas. En éste se exponen visualmente, las zonas geográficas de visibilidad y penumbras a las radiaciones de fuentes electromagnéticas puntuales. En el desarrollo del ejercicio se contemplan dos aspectos: el primero elabora un modelo geométrico en 3D que represente el perfil topográfico de regiones geográficas, a partir de las bases de datos disponibles como resultado del proyecto SRTM (NGA and NASA) [8]. El segundo, propone un modelo de propagación para una fuente de radiación ondulatoria en el espacio libre de cargas y fuentes en regiones homogéneas e isotrópicas, de acuerdo con la solución de Maxwell a la propagación de ondas electromagnéticas y en concordancia con la ley de Snell y las técnicas del Ray-Tracing (RT).

1 Grupo de Investigación en Mecánica Aplicada Universidad Eafit. E-mail: jairo.madrigal@gmail.com.

- Grupo de Investigación en Mecánica Aplicada Universidad Eafit. E-mail:mgarcia@eafit.edu.co 


\section{Palabras clave}

Trazado de rayos, Red de elementos triangulares, Oclusión visual, Solución a la ecuación de onda.

\section{Abstract}

This article exhibits a computational procedure that allows, on a Terrain Elevation Map (TEM) of a geographic region of interest, areas that are in line with direct impact of energy flows generated from point sources located. This presents visually, the geographical areas of visibility and penumbras of electromagnetic radiation of punctual sources. In developing of the exercise have been covered two aspects: the first produces a $3 \mathrm{D}$ geometric model that represents the topographic profile of geographical regions, based on the available databases as a result of the project SRTM (NGA and NASA) [8]. The second, proposed a model of propagation for a radiation source wave in the space free of charge and sources in homogeneous and isotropic regions, according to the solution of Maxwell to the propagation of electromagnetic waves and in accordance with the law and Snell and techniques of Ray-Tracing (RT).

Key words

Ray tracing, Triangulated Irregular Network, Oclusión visual, Wave ecuation solution. 


\section{INTRODucción}

En el estudio de la propagación de las ondas electromagnéticas se han planeado modelos entre los que aparece la teoría electromagnética de Maxwell, la teoría de difracción de Kirchhoff y la óptica geométrica. Los modelos generalizados de propagación de ondas electromagnéticas, sean teóricos o empíricos, son diversos, y dependen de la complejidad del modelo que se desea implementar. En el espacio real, las soluciones de Maxwell compatibles con las condiciones de contorno que imponen los medios de manera rigurosa, hacen de este estudio un ejercicio a veces impracticable y en todo caso, muy complejo. La información que se les exige procesar a los modelos geométricos utilizados requieren del conocimiento de la distribución espacial de los elementos que intervienen en el escenario, la disposición y dimensión geométrica de los objetos que pueden interactuar sobre el pulso entre la fuente y la región de propagación y que pueden actuar como agentes dispersores, reflectores y/o difractores a las señales electromagnéticas.

El modelo de Okumura-Hata $(\mathrm{OH})$ [4], contiene una formulación empírica basado en mediciones sobre el terreno para calcular las pérdidas de propagación en zonas urbanas en señales de frecuencias entre 150 y $1500 \mathrm{MHz}$, para antenas isotrópicas, entre 30 y $200 \mathrm{~m}$ de altura efectiva y antenas móviles entre 1 y $10 \mathrm{~m}$ de altura con separación entre 1 y $20 \mathrm{~km}$ [1][2].

El modelo COST 231 Walfisch-Ikegami [1], [5] es un rápido predictor empírico para escenarios urbanos en el campo de las comunicaciones móviles referenciado al ambiente europeo. Perfilado por la organización COST 231 por los resultados obtenidos.

El modelo Longley-Rice (LR)[6] es un algoritmo de computadora que predice las pérdidas relativas al espacio libre. Requiere parámetros como la frecuencia de transmisión, alturas entre las antenas receptoras y transmisoras, distancia entre las antenas, reflectividad media de las superficies, valores de la constante de la conductividad de la tierra, polarización vertical y horizontal, y descripción de la tierra. Este modelo es de uso corriente para 
frecuencias entre $20 \mathrm{MHz}$ y $20 \mathrm{GHz}$ y para antenas con alturas entre 0.5 y $300 \mathrm{~m}$. con una separación entre antenas TR (TransmisorReceptor) de 1 a $2000 \mathrm{~km}$.

El modelo Terrain Integrated Rough Earth Model (TIREM) [4], desarrollado por la Oficina del Departamento de Comercio, Telecomunicaciones Nacionales y Administración de Información de los Estados Unidos, predice pérdidas en propagación entre dos puntos considerando la frecuencia de transmisión, y parámetros de transmisión atmosférica, tales como permitividad y conductividad de la tierra, los perfiles del terreno entre el transmisor y el receptor. Validado para frecuencias entre $40 \mathrm{MHz}$ y $20 \mathrm{GHz}$.

El modelo de predicción CRC [4], desarrollado por el Centro de Investigaciones en Comunicaciones, en Ottawa, calcula las pérdidas debido a la difracción, usando información sobre el terreno y cubrimiento de las señales radioeléctricas. De uso corriente en el rango de frecuencias entre 30 y $3000 \mathrm{MHz}$, alturas de las antenas transmisoras entre 30 y $200 \mathrm{~m}$ y receptoras entre 1 y $10 \mathrm{~m}$.

La implementación de estos modelos cuenta con gran disponibilidad de documentación y códigos fuentes, más orientados en el tema de la propagación móvil, aunque el volumen varía entre los modelos, el grado de desarrollo y los costos. Los modelos más referenciados enfatizan en la cobertura zonal, la multiplicidad de trayectos entre el transmisor y receptor, la variabilidad de los trayectos y el nivel de la señal recibida [4].

En el procedimiento aquí expuesto se sitúa una fuente puntual de radiación de onda esférica sobre la red de elementos triangulares construida sobre el valle del Aburrá. Calcula la interceptación del frente de onda sobre cada uno de los elementos triangulares de la red, de esta manera se determinan las zonas expuestas a la incidencia directa y las zonas de oscuridad a la radiación. Este modelo, permite calcular mediante el teorema de Poynting, la intensidad del campo de propagación de pulsos, en un rango amplio de longitudes de onda, limitado a radiaciones de fase espacial del mismo orden de la resolución de la celda unitaria del elemento triangular de la que modela la red. Este artículo presenta el modelo 
geométrico del terreno, clasifica los elementos triangulares de la red que están en la línea visual en exposición directa y los que son susceptibles de ser expuestos por reflexión. De esta manera, es posible construir mapas de visibilidad y penumbras a la radiación de fuentes puntuales.

\section{WODKLADO CEOMETRICO DE TERENO}

En el modelado geométrico del terreno, comúnmente llamados mapas digitales del terreno DTM y DEM, acrónimo del inglés Digital Elevation Model, se hacen representaciones abstractas de propiedades localizadas sobre el terreno, limitados no sólo por las capacidades computacionales sino por la complejidad de los modelos matemáticos de las propiedades que se desean representar. Los sistemas de información geográfica, GIS, ofrecen en diferentes sistemas, coordenados en variedad de formatos, aspectos referentes a la topografía y propiedades de las regiones censadas satelitalmente.

El modelo en representación del valle del Aburrá ha sido adquirido de los datos procesados por Suttle Radar Thopography Mission SRTM [5], en formato matricial de filas y columnas [m,n], muestreados a una resolución de un segundo de arco, correspondiente a una longitud espacial de $30 \mathrm{~m}$ sobre la superficie terrestre. En este modelado, el número asociado con cada coordenada muestral es su elevación medida en metros. En aras de simplificar los cálculos usados en este procedimiento, se ha estimando que la representación numérica de los datos adquiridos de la base de datos no contiene distorsiones y que las mediciones no han sido afectadas significativamente por parámetros, tales como efectos de sombreado, dispersión y/o difracción, gradientes de temperatura, entre otros.

La región correspondiente al valle del Aburrá está ubicada geográficamente en el rango de coordenadas en la zona: lat: 6.133347 a 6.359292 y long: 2.322182 a 2.466188 . En la figura 1 se muestra el modelo de elevación digital de esta zona; allí son 
observables el perfil topográfico y rasgos, tales como regiones planas, lomas, picos, entre otros.

Figura 1. Modelado de la distribución topográfica del valle del AburRá

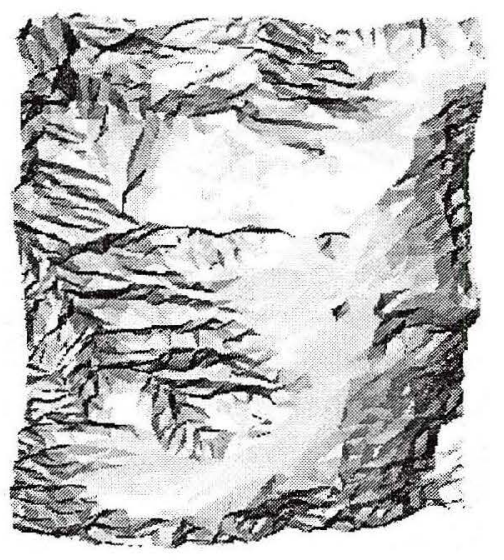

Para la representación geométrica de la superficie, se arregla el conjunto finito de puntos

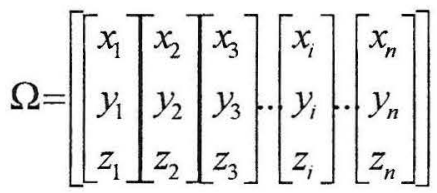

$$
\begin{aligned}
& \operatorname{Con} \Omega\left(:, v_{i k}\right)=\left[\begin{array}{l}
x_{k} \\
y_{k} \\
z_{k}
\end{array}\right]
\end{aligned}
$$

La expresión (1.1) representa las coordenadas cartesianas en $3 \mathrm{D}$ del punto $k$ de la malla, donde los puntos de elevación son asociadas a las componentes $z_{k}$. El arreglo expresado de la forma (2) representa el conjunto de trío de puntos contiguos que, define cada uno los vértices de un elemento triangular de la superficie, a partir de los cuales se construyen celdas unitarias triangulares, 


$$
\Delta_{k}=\left\lfloor v_{1 k} v_{2 k} v_{3 k}\right\rfloor
$$

Los valores $1_{k}, 2_{k}, 3_{k}$, corresponden a los vértices $1,2,3$, de la celda unitaria $k$ de un punto coordenado en $\Omega$. La celda unitaria es ilustrada en la Figura 2, y es la base de la construcción del enmallado,

$$
v_{i}=\left[\begin{array}{lll}
x_{i} & y_{i} & z_{i}
\end{array}\right]
$$

Figura 2. Celda unitaria de la malla

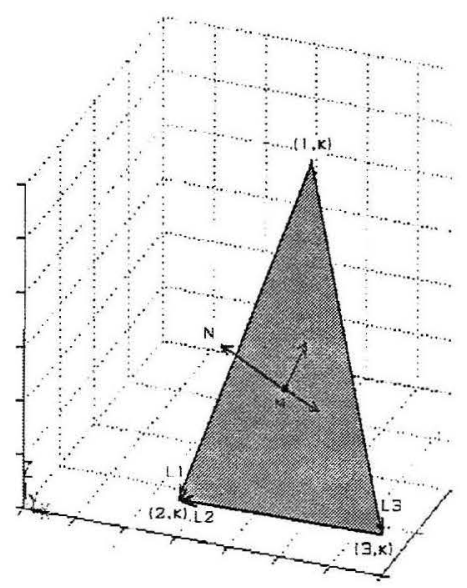

Mediante técnicas de interpolación de superficies asociadas a funciones base, la totalidad del terreno, entonces, se verá representada por un modelo de conexión entre los puntos en una red de elementos triangulares irregulares TIN (Triangulated Irregular Network), ubicando en un espacio cartesiano los puntos que marcan su posición geográfica; y el valor calculado para la propiedad en estudio en cada punto muestreado por el satélite. La Figura 3 ilustra la red que representa el valle del Aburrá, en ésta es posible ver que la no regularidad en la distancia entre puntos es una consecuencia de irregularidades en el terreno, 
Figura 3. TIN SOBRE EL VALLE del AburRÁ

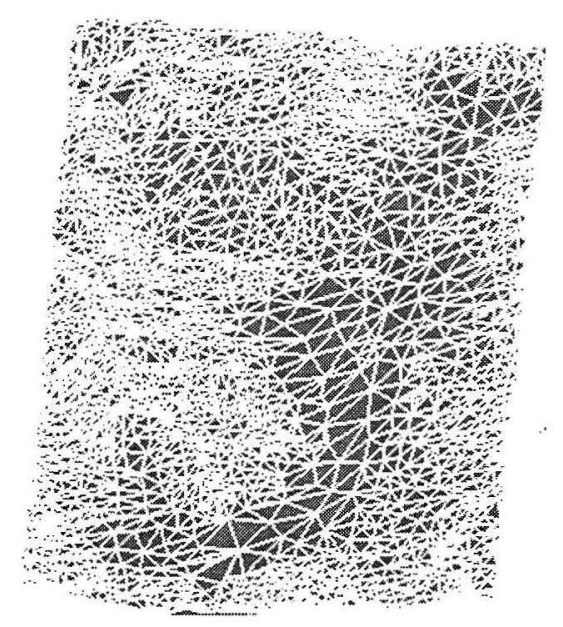

siendo menos densas las regiones de relieve suaves o planas. Los elementos constitutivos de los DEM, generalmente son ordenados matricialmente, proporcionando facilidad en la estructura de la manipulación de los datos.

\section{OCLUSION UISUAL}

Dos condiciones deben satisfacer los elementos triangulares de la TIN, para ser clasificados dentro de la línea de visibilidad de la fuente. La primera de ellas es la llamada propiedad de coherencia, en la cual el vector normal de la cara del elemento triangular, está orientado en sentido opuesto al vector que une el punto donde se encuentra la fuente con el baricentro del elemento triangular, ésta es condición de la visibilidad [6]. La segunda, es establecida mediante un test de visibilidad en el cual, se definen cuáles de los elementos triangulares que satisfacen el test de coherencia en un mismo rayo de visión, es el más cercano a la fuente, ya que el más lejano está eclipsado por el más cercano. 
Entonces, se propone un modelo de rayo para la propagación de los pulsos ondulatorios, irradiados por la fuente en supuestos medios homogéneos e isotrópicos, donde las trayectorias de propagación de los frentes de onda son rectilíneos en la dirección en la que fluye la energía; para el caso de una onda esférica, la dirección de propagación será radialmente alejándose de la fuente.

Estas condiciones son tratadas con los procedimientos del Ray Tracing (RT), fundamentado en la geometría vectorial. Requiere de la determinación de la posición de la fuente, el lugar del elemento de la TIN donde está ubicado el receptor, además de su configuración espacial. La condición de coherencia usa la propiedad del producto punto entre el vector normal del elemento triangular $\Delta_{k}$, con el vector de incidencia directa (RD), seleccionando los que forman ángulos agudos.

Sólo para $\theta \leq \pi / 2$

La condición (4) es establecida como el test de anti paralelismo y el valor del ángulo puede ser calculado mediante la ecuación (5):

$$
\theta=\cos ^{-1}\left(\frac{R \vec{D}_{i} \bullet \vec{N}_{i}}{\left|R \vec{D}_{i}\right|\left|\vec{N}_{i}\right|}\right)
$$

Este procedimiento contempla aspectos como la determinación de la superficie triangular en estudio. Para el elemento triangular $\Delta_{k}$ dado por la conexión nodal expresada en la ecuación (2), se extraen los tres lados del triángulo calculando las coordenadas cartesianas del vértice $i$ del elemento triangular $k$.

Así los segmentos de recta $\mathrm{L}_{1}, \mathrm{~L}_{2}, \mathrm{I}_{3}$

$$
\begin{aligned}
& \vec{L}_{1}=\left[\Omega\left(:, v_{1 k}\right)-\Omega\left(:, v_{2 k}\right)\right] \\
& \vec{L}_{2}=\left[\Omega\left(:, v_{3 k}\right)-\Omega\left(:, v_{2 k}\right)\right] \\
& \vec{L}_{3}=\left[\Omega\left(:, v_{1 k}\right)-\Omega\left(:, v_{3 k}\right)\right]
\end{aligned}
$$


El baricentro del triángulo se puede calcular asumiendo el mismo factor del peso para cada uno de los puntos:

$$
M_{k}=\frac{1}{3} \sum_{i=1}^{3} v_{i k}
$$

$M_{k}$ contiene las coordenadas del baricentro del elemento $\Delta_{k}$

El vector normal de $\Delta_{k}$ se calcula con base en las propiedades del producto cruz entre los vectores $\vec{L}_{i}, \vec{L}_{j}, \vec{L}_{k}$ que definen los lados del triángulo. Esto, si los lados del triángulo están arreglados acorde con la convención de la mano derecha,

$$
\vec{N}_{k}=\vec{L}_{2} \times \vec{L}_{1}
$$

El test de visibilidad define de esta manera cuáles de los elementos por los que cruza el rayo de visión es el más cercano a la fuente.

Si $r_{s}$ es la posición de la fuente y $M_{i}$ es la posición del receptor, el RD de puede definir mediante la relación:

$$
R \vec{D}_{i}=M_{i}-r_{s}
$$

Así para cada $\Delta_{i}$ hasta el total del barrido

$$
V=\left[V, R \vec{D}_{i}\right]
$$

Recoge finalmente el menor de los elementos de $V$ y se compara con la magnitud del rayo directo a $D R_{k}$

$$
\text { Delta }=|R \vec{D}|_{k}-\left|R \vec{D}_{i}\right|
$$

El test selecciona aquéllos que satisfacen la condición de los elementos que cruza el RD, cuál es el más cercano a la fuente. Esta condición se satisface para los valores Delta menores que cero. 
En consecuencia, aquellos elementos que satisfacen el test de visibilidad serán susceptibles de ser alcanzados por la exposición directa a las fuentes de radiación. En la Figura 4 se exhiben las zonas que están dentro del campo visual, en ésta las zonas oscuras corresponden a zonas de oscuridad a la radiación de la fuente puntual localizada. En este modelo las características de la fuente no son relevantes ya que el valor de la intensidad, no tiene peso para este test.

\section{Figura 4. dem asociado a LA ocLusión Visual sobre el VAlle del AburRá DEBIDO A UNA FUENTE PUNTUAL LOCALIZADA}

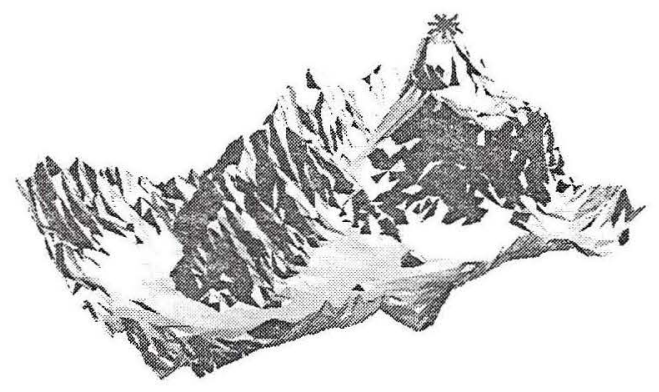

\section{H. CANPO REFLEDAOO}

En la realización de un estimativo de las zonas expuesta a la radiación por reflexión, se ha considerado un modelo de reflexión especular, de acuerdo con las leyes de Snell y los postulados de la óptica geométrica [3]. Este procedimiento es similar al filtrado que establece la oclusión visual, tomando sólo el grupo de los elementos que han sido expuestos a la incidencia directa al RD desde la fuente. En este procedimiento cada uno de los $\Delta_{k}$, que ha aprobado el test de oclusión visual, se ha considerado una nueva fuente puntual de radiación, se realiza un test de antiparalelismo y oclusión para el Rayo Reflejado (RR) y, de esta manera, se calcula los elementos de la TIN que se exponen a los campos debido a la reflexión especular sobre los elementos triangulares. Para el cálculo 
del RR basta con descomponer el RD en sus componentes paralela y perpendicular con respecto al plano de la celda, y reordenar estas componentes de manera que satisfagan la ley de Snell, para reflexión especular. En el momento de calcular el vector $R R$ basta con tomar la componente paralela, sin cambios, en tanto que la componente perpendicular cambia de dirección en 180 grados. El vector RR tendrá su origen en el baricentro, para fines prácticos, ver Figura 5. El procedimiento para el cálculo del vector $R R$ puede escribirse de la siguiente manera, sean:

$r_{s}$ : las coordenadas cartesianas de la fuente de puntual y $M_{i}$ las coordenadas baricéntricas del elemento $i$. $N_{i}$ es el vector normal del elemento $i$ calculado mediante la ecuación (8). La componente perpendicular de RD es calculada usando la relación con la normal del triangulo, ecuación (12).

$$
\vec{R}_{D \perp}=\left(\vec{N}_{i} \bullet \vec{R} D\right) \vec{N}_{i}
$$

La componente $\mathrm{RD}$ paralela a la cara se calcula restando la componente perpendicular, ecuación (13)

$$
\vec{R}_{D \prod}=\vec{R}_{D}-\vec{R}_{D \perp}
$$

Así el rayo reflejado se calcula sumando las componentes paralela con la componente perpendicular en dirección contraria, cuyo origen en el baricentro del triángulo $\Delta_{i}$ como se ilustra en la ecuación (14)

$$
\vec{R}_{R}=M_{i}+\vec{R}_{D \perp}-\vec{R}_{D \Pi}
$$

Luego de ser calculados los elementos triangulares susceptibles de ser expuestos por las reflexiones especulares de los múltiples elementos constitutivos de la red, se les calcula la intensidad del campo de radiación. 
La Figura 6 muestra de acuerdo con la intensidad del color en escala de grises, la variabilidad de la intensidad sobre los elementos triangulares expuestos a los campos reflejados cuyas fuentes de radiación han sido los elementos triangulares alcanzados por la exposición directa a la fuente.

Figura 5. Rayo Directo (RD) y Rayo Reflejado (RR)

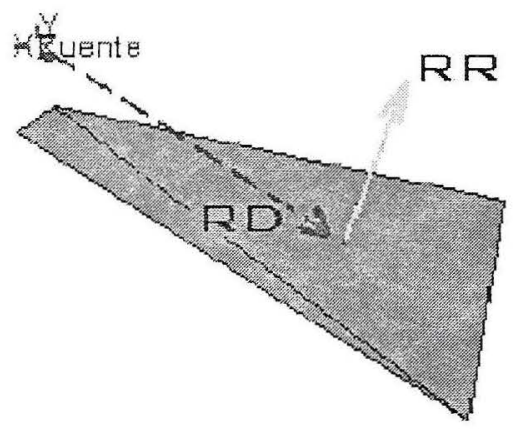

Figura 6. EXHIBE LOS ELEMENTOS DE LA TIN EXPUESTOS A LAS REFLEXIONES DE LAS CELDAS VECINAS

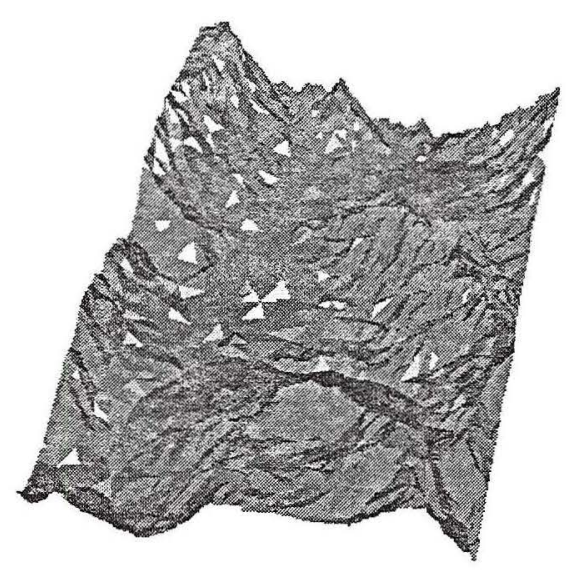




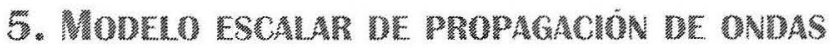

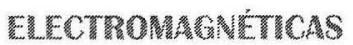

El modelo de propagación de ondas electromagnéticas en el espacio libre ha sido descrito ampliamente en el campo de la teoría electromagnética de Maxwell, que propone como solución al problema de la propagación de los campos eléctricos y magnéticos la ecuación de ondas [3], [7]:

$$
\nabla^{2} E(r, t)-\frac{1}{C^{2}} \frac{\partial^{2}}{\partial t^{2}} E(r, t)=0
$$

Siendo $E(r, t)$ la forma de expresar el campo eléctrico en función de la posición y el tiempo.

La solución particular para la ecuación (15) $E(r, t)$ depende del tipo de onda que desea modelar, sea un modelo de onda plana, onda esférica u onda cilíndrica y del sistema de coordenadas en el cual se haga la representación.

El modelo de función solución, para ecuación de ondas (15) [3], generada por una fuente puntual que radia esféricamente, puede escribirse en la forma de la ecuación (16)

$$
F(r, t, \phi)=\frac{E_{0}}{r} \operatorname{sen}(\vec{k} \bullet \vec{r}-w t+\varphi)
$$

En la ecuación (16) $E_{0}$ representa la amplitud del campo eléctrico en el lugar de la fuente de radiación, y $F(r, t, \phi)$ representa la amplitud de campo eléctrico en el lugar en el punto r. $w=$ $2 \pi f$ es la frecuencia temporal, $\lambda$ la longitud de onda, $k=2 \pi / \lambda$ la frecuencia espacial, $c=\lambda f$ velocidad de propagación del pulso y $\varphi$ es el desplazamiento de la fase espacial.

$$
\vec{r}=\vec{r}_{s}-\vec{r}_{i}
$$

La ecuación (17) representa el vector que asocia la posición de la fuente y el punto de incidencia de la onda $\mathrm{RD}$, de interés para el 
cálculo del valor de la función. La Figura 7 muestra tres perfiles de ondas que son soluciones a la ecuación de onda (15).

Figura 7. Perfiles de pulsos en $2 D$ y $3 D$

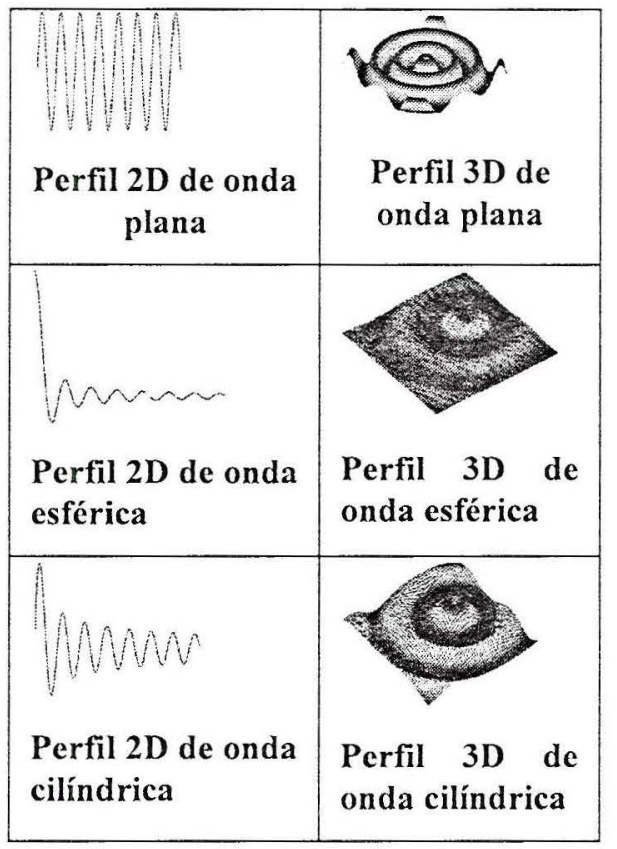

En la Figura 8, la distribución de los colores indica los niveles de intensidad del campo de radiación sobre el terreno, de acuerdo con el perfil de la onda esférica de la Figura 7. En esta figura pueden observarse tanto los niveles de intensidad del pulso como las regiones de la TIN, que son alcanzadas por exposición directa a la fuente; las zonas que no están coloreadas son las zonas de oscuridad para la fuente localizada en el punto más alto de la región del valle del Aburrá y, por lo tanto, son ciegas para la fuente. 
Figura 8. Distribución de onda esférica ocluida sobre La tin del Valle del AburRá

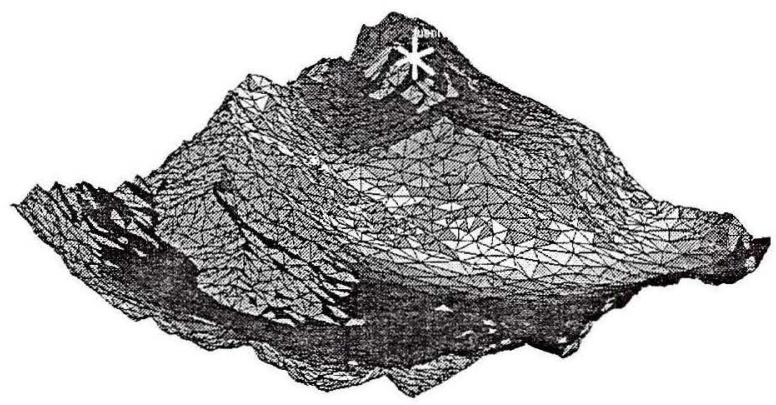

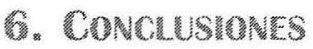

En el espacio real, las soluciones de Maxwell compatibles con las condiciones de contorno, que imponen los medios de manera rigurosa hacen de este estudio un ejercicio a veces impracticable y en todo caso, muy complejo. En el sentido práctico, cuando los modelos teóricos dejan de ser válidos, se acuden a modelos empíricos o a curvas de propagación normalizadas, a partix de mediciones realizadas en el terreno para optimizar los modelos teóricos cuando se requiere buena precisión.

El modelo aquí expuesto, basado en la óptica geométrica y en un modelo de propagación de ondas electromagnéticas simplificado; resulta de utilidad práctica en la predicción de zonas de visibilidad y oscuridad ante una fuente puntual de generación de energía electromagnética, cuando se trata de obtener rapidez en la ejecución y en la elaboración de los procedimientos que dan respuestas de utilidad práctica en el diseño de topologías de redes de receptores y transmisores.

Aunque las técnicas del Ray Tracing ( $\mathrm{R} T$ ) son generalmente usadas en la reproducción de escenarios, y en la exaltación de detalles donde la resolución espacial es del orden de la longitud de onda del rango visible del espectro electromagnético; sus procedimientos resultan en esta aplicación, de utilidad en el rango de las radiofrecuencias; y puede generalizarse a frecuencias espaciales 
acotadas por la resolución espacial de los elementos poliédricos de la red de que describen las regiones de aplicación.

Como aplicación práctica, esta herramienta computacional puede ser usada por los científicos e ingenieros del gremio de las telecomunicaciones, para efectos de la proyección de las coberturas de los sistemas de comunicación y diseño de topologías de redes de estaciones de transmisión y/o repetidoras. También, puede ser útil como instrumento pedagógico para la representación de los modelos de propagación ondulatoria, usados en el ambiente de los sistemas de telecomunicaciones y de la óptica geométrica; o por estudiantes en el desarrollo de modelos de fenómenos físicos que tienen que ver con la óptica ondulatoria, la óptica geométrica y la propagación de flujos mecánicos y electromagnéticos.

Este ejercicio ofrece un acercamiento al tema de la propagación de ondas electromagnéticas, quedando en perspectiva, la posibilidad a futuro de aplicación de modelos de propagación más complejos sobre regiones geográficas.

\section{Traranos chanos}

[1] COST-231 Walfisch-Ikegam. (1988). Obtenido de A fast empirical prediction model for urbans scenarios: http://www.awe-communications. com/Propagation/Urbans/COST/index.htm

[2] Hernando Rábanos, J. M. (2004). Comunicaciones móviles. Madrid: Editorial universitaria Ramón Areces.

[3] Hetch, E. (2005). Óptica (3 ed.). Ed. Pearson, Addison Wesley.

[4] Hogan, M. K. (2005). Advanced Mission Planning Tool for Real-Time Kinematic (RTK) GPS Surveying. San Diego: ION Meeting Proceedings.

[5] Lee, J. S. (1998). CDMA Systems Engineering Handbook. Boston: Artech House publishers.

[6] Longley, A. G., \& Rice, P. L. (1968). Prediction of tropospheric radio transmission over irregular terrain, a computer method-1968. Washington, DC: ESSA technical report. ERL 79-ITS 67, U.S.

[7] Marion, J. B. (1980). Classical Electromagnetic Radiation (Second ed.). Academic Press. 
[8] NGA and NASA. (2006). Obtenido de The Shuttle Radar Topography Mission (SRTM). Project Formulation \& Management Applications (PFMA) Group: http://www2.jpl.nasa.gov/srtm 